\title{
Helicobacter pylori: continuing challenges
}

Coelho LGV. Helicobacter pylori: continuing challenges. Arq Gastroenterol. 2018;55(2):95-6.

This edition contemplates two articles about different aspects on Helicobacter pylori (HP) infection: the daily medical practice challenges on its management and the search for comprehension of still obscure points among functional interaction of the infection and human immune system, on the development of gastroduodenal diseases ${ }^{(1,2)}$.

On the first paper, the Núcleo Brasileiro para Estudo do Helicobacter pylori e Microbiota (NBEHPM), association linked to Brazilian Federation of Gastroenterology (FBG), founded on 1994, presents its IV $^{\text {th }}$ Brazilian Consensus Conference on Helicobacter pylori infection, composed by 30 statements and divided into four groups as follows: 1) Epidemiology and Diagnosis, 2) Dyspepsia, intestinal microbiota and other affections, 3) Gastric Cancer, and, 4) Treatment.

The first group shows that Brazilian H. pylori epidemiology is similar to other areas of the emerging world, with high prevalence rates of HP infection in urban and rural regions, with unhealthy environments and precarious life conditions during childhood. In contrast, richer urban healthy populations, children whose parents were more educated, families with a better socioeconomic status, and individuals living in cities with better sanitation and housing conditions demonstrated that the prevalence rates of HP infection tends be lower. Despite being the worldwide gold standard test for non-invasive diagnosis of HP infection, the 13C-urea breath test (UBT) has not yet been incorporated into daily gastroenterological practice in Brazil, and its availability remains restricted to large cities and university centers. It is emphasized the need toward improving the availability of the UBT throughout the country, which will positively influence the diagnosis and therapeutic management of HP. Still regarding HP diagnosis, it is important to disseminate the use of genotypic method to identify point mutations responsible for HP resistance to antimicrobials such as clarythromicin, levofloxacin, and tetracycline. Such methodology is now regularly available using molecular biology techniques and could be centralized in some centers all over the country. This could partially substitute microbiological techniques not easily available on endoscopy rooms.

On dyspepsia, microbiota and other conditions statements the authors adopted the latest decision of two international guidelines (Kyoto Consensus Conference and Rome Consensus IV) to classify HP-associated dyspepsia, not as functional but as a distinct type of organic dyspepsia in which symptoms can improve steadily after bacterial eradication (6 to 12 months). In this way, all patients with dyspepsia and HP should be submitted to eradication treatment. The role of human intestinal microbiota was also reviewed. Classically thought to be a sterile organ, due to its acid production, the release of bile acids by the liver, the constant peristalsis of gastric mucosa, and the presence of nitric oxide (antimicrobial properties), the human stomach is now considered to hold a core microbiome in addition to HP. Initial studies using $16 \mathrm{~S}$ rDNA identified more than 120 distinct bacterial phylotypes on the gastric mucosa of healthy individuals, mainly Actinobacteria, Bacterioidetes, Firmicutes, Fusobacteria, and Proteobacteria ${ }^{(3)}$. The lost of stomach acid environment caused by diseases such as autoimune gastritis and HP infecction or use of antisecretory drugs (PPI, histamine $\mathrm{H}_{2}$-receptor antagonists) can raise the risk of gastric colonization by microorganisms and impacts on gastric physiology and function. With two related statements, this Brazilian Consensus considered premature the recommendation of prebiotics, probiotics, and symbiotics as an attempt to optimize HP eradication and minimize adverse events.

Gastric neoplasias group confirmed that Brazil has an intermediate incidence of gastric adenocarcinoma, with regional differences and higher frequency in the north and northeast compared with southeast region. Clinical, epidemiological, and molecular studies clearly show that HP infection is the major etiological factor related to gastric adenocarcinoma. It is estimated that nearly $80 \%$ of gastric malignant tumors are associated to HP infection and the risk of gastric adenocarcinoma is similar in the diffuse and intestinal subtypes. Level $1 \mathrm{~A}$ of evidence statement demonstrate that $\mathrm{HP}$ eradication is associated with a decrease in the rate of gastric adenocarcinoma. This reduction is shown when the eradication occurs before the establishment of pre-neoplastic lesions, although could be still partially effective in those with established pre-neoplastic lesions, and potentially effective to prevent metachronous gastric cancer. Regarding gastric adenocarcinoma secondary prevention, histological staging of gastritis (OLGA or OLGIM system) is recommended based in recent studies and as well in expert opinion is recommended that patients staged as OLGA III or IV should undergo endoscopic follow-up every two years.

Last but not least, the Consensus Conference approaches different aspects on anti- $H$ pylori treatment, taking into account the raising rates of antibiotic resistance observed worldwide including Brazil. Regarding clarithromycin, key-antibiotic to first-line therapy against HP, some national studies have shown rates of primary $H$. pylori clarithromycin resistance situated in the borderland (15\%-20\%) where clarithromycin-containing triple therapies are still allowed to be empirically used. One challenging point in Brazil is the consumption of antibiotics by the population. Previous studies from Europe have shown that the antibiotic consumption in a given region, may provide a simple tool to predict the susceptibility of H. pylori to quinolones and macrolides in the population. There are data showing that the selling of macrolides (mostly azithromycin) in Brazil jumped from 12.5 millions of units/year in 2008 to 21.5 millions of units/year in $2017^{(4)}$. Besides that, the very low cost and the indiscriminate use of azithromycin by the population can contribute to promote the increase of clarithromycin resist- 
ance in near future. It is well recognized that azithromycin can achieve high concentrations in the gastric mucus and gastric juice for several weeks after its administration, and this may lead to local sub-inhibitory concentrations and select macrolide-resistant mutants. Classical triple therapy now extended for 14 days remains the first-line therapy recommendation by the group. First-line alternative therapeutic regimens, rescue therapies and therapy to penicillin-allergic patients are well described in the guideline.

The 30 statements of this Consensus Conference are intended to provide an updated best-practice for $H$. pylori infection management in Brazil. Additional studies should focus on assessing antibiotic resistance rates across Brazil to ensure resistance patterns in the population. In addition, high quality studies evaluating novel approaches and therapies including bismuth-based regimens, quadruple concomitant therapy, and the role of supplemental probiotics are needed.

Another stimulating article published in this issue from Arquivos de Gastroenterologia, comes from Para State, in the North of Brazil, where researchers evaluated the immune response (bacterial genotype and cytokine production) induced by $H$ pylori infection among 506 infected patients with gastritis $(n=244)$, gastric ulcer $(n=51)$, duodenal ulcer $(n=66)$ and gastric cancer $(n=145)$. All patients studied came from the same region, had similar culture habits, socio economic level, and ethnic background. The main virulent factors of $H$. pylori, vacuolating cytoxin A (VacA) and cytotoxin-associated gene A (CagA), were found in more than $85 \%$ of the strains, with patients with gastric ulcer, duodenal ulcer and gastric cancer ex- hibiting higher frequency of the virulent s1m1cagA genotype than patients with only gastritis. Regarding the cytokine concentrations in the gastric mucosa it was found that patients with gastric ulcer and gastric cancer presented higher levels of IFN- $\gamma$ and IL-12 and lower levels of IL-4 and IL-10 compared with patients with gastritis and duodenal ulcer. In addition, patients harbouring s $1 \mathrm{~m} 1 \mathrm{cag} A$ virulent strains showed also higher concentrations of INF- $\gamma$ and IL-12. It was demonstrated that patients infected with s1mlcagA+ strains had higher degree of inflammation, neutrophil activity and increased risk of developing intestinal metaplasia. These findings confirmed previous studies indicating that an increased inflammatory response, particularly linked with an over-production of pro-inflammatory cytokines and mediators in the Th1 pathway, increase the odds ratio of $H$. pylori-positive individuals developing more serious diseases, especially gastric cancer ${ }^{(5)}$. In the last two decades, there has been a remarkable progress in identifying and understanding the multitude of microbes that colonize the human body ${ }^{(6)}$. With the improvement of sequencing technology, the possibility to analyze microbiota throughout the whole GI tract independently of cultural techniques will allow a great advance of our knowledge on gastro-duodenal microbiota, especially about $H$ pylori interactions with gastric microbiota and immune response on gastroduodenal disorders. Further studies may clarify in what extension the gastric microbiota can modulate the immune response of the host and the outcome of an HP infection.

Luiz Gonzaga Vaz COELHO*

\section{REFERENCES}

1. Coelho LGV, Marinho JR, Genta R, Ribeiro LT, Passos MCF, Zaterka S, et al. IVth Brazilian Consensus Conference on Helicobacter pylori infection. Arq Gastroenterol. 2018;55(2):97-121.

2. Vinagre RMDF, Vinagre IDF, Vilar e Silva A, Fecury AA, Martins LC. Helicobacter pylori infection and immune profile of patients with different gastroduodenal diseases. Arq Gastroenterol. 2018;55(2):122-7.

3. Bik EM, Eckburg PB, Gill SR, Nelson KE, Purdom EA, Francois F, Perez-Perez G, Blaser MJ, Relman DA. Molecular analysis of the bacterial microbiota in the human stomach. Proc Natl Acad Sci USA. 2006;103:732-7.

4. IQVIA (IMS Health) - Pharmaceutical Market Brasil (2008-2017).

5. El-Omar EM. The importance of interleukin $1 \beta$ in Helicobacter pylori associated disease. Gut. 2001;48:743-7.

6. Nardone G, Compare D. The human gastric microbiota: Is it time to rethink the pathogenesis of stomach diseases? United Eur Gastroenterol J. 2015;3:255-60.

\section{(cC) BY-NC}

